\title{
Auto-monitoring: theoretical touchstone or circular catch-all?
}

\author{
Nick Hammond
}

\author{
CTI Centre for Psychology, University of York, UK
}

Until I read Ray McAleese's paper, I perhaps had a rather simplistic psychologist's view of concept mapping. It was, I felt, a technique - one of several - that helped learners to articulate their burgeoning understanding of some topic, providing a canvas on which to record, expand and manipulate their knowledge. The activities involved in concept mapping could be linked to well-known psychological principles of understanding, memorization and learning: effort, elaboration and depth of processing; generation and enactment effects; encoding specificity and encoding variability; the distinctions between explicit and implicit representations; metacognitive strategies and reflection, and so forth (Hammond 1993). These psychological underpinnings, while not in any sense providing an integrated 'theory' of concept mapping, give a view of when and why the use of concept mapping might be effective in some situations and not in others, and how different concept mapping tools differ in the claims they are making about their educational use (Trapp, Reader and Hammond 1992).

The concept of auto-monitoring provides a related but higher-level perspective. Thus, as McAleese argues, it links the areas of reflection, meta-cognition and concept mapping. It also provides a process view of the stages involved in concept mapping (McAleese, Figure 2). There is perhaps some theoretical leverage here. (McAleese wisely steers clear of the tempting but fallacious argument that maps are good for leaming because they reflect structures in the head in some way - this fallacy is analysed by McKendree and Reader 1994.) However, McAleese is claiming more than this: it is not just that concept mapping can be a rather effective way of learning because it requires explication and reflection; the claim (as I understand it) is that concept mapping helps students develop auto-monitoring techniques and so enhance their critical thinking skills. While acknowledging that this might be the case, I have yet to see either any evidence or a persuasive theoretical argument that supports it. There have, after all, been many contenders for this educational equivalent of the philosopher's stone, from Latin grammar to Logo programming. Whether or not concept mapping does assist in the development of thinking skills (over and above providing a specific technique for helping lay out one's knowledge) is still an open empirical question.

It is here that I have a slightly uncomfortable feeling of circularity. To simplify the argument: critical thinking skills depend substantially on reflection, self-monitoring and gaining an overview, in short on auto-monitoring; concept mapping is a set of activities which both requires and develops auto-monitoring; therefore concept mapping develops critical thinking skills. But is the concept of auto-monitoring as yet sufficiently well-defined or robust to serve as the bridge? The flaw is that none of these three concepts (thinking skills, auto-monitoring or concept mapping) is unitary, and the links between them are strictly hypothetical. Thinking involves a complex amalgam of different skills, which have to be appropriately mobilized (implicitly or explicitly), depending on the situation. As McAleese points out, auto-monitoring as an 'executive control' is likely to involve a range of mental procedures. And concept 
mapping supports a diverse range of learning and comprehension tasks. The hypothesis is therefore a challenging one, though not yet proven. But perhaps I have gone further than McAleese is claiming. I am certainly happy with his view of auto-monitoring as a "learning arena' in which the learner can be aware of and manipulate learning activities.

\title{
References
}

Hammond, N.V. (1993), 'Learning with hypertext: problems, principles and prospects', in McKnight, C., Dillon, A. and Richardson, J. (eds), Hypertext: A Psychological Perspective, Chichester, Ellis Horwood.

-McKendree, J. and Reader, W. (1994). "The "homeopathic fallacy" in hypertext: misconceptions of psychology in design of computer courseware', ALT News (in press).

Trapp, A.L., Reader, W. and Hammond, N.V. (1992), 'Tools for knowledge mapping: a framework for understanding' in Brusilovsky, P. and Stefanuk; V. (eds), East-West Conference on Emerging Technologies in Education, Moscow, April.

\section{Concept mapping and other formalisms as Mindtools for representing knowledge}

\author{
David H. Jonassen* and Rose M. Marra** \\ ${ }^{\star}$ Pennsylvania State University and ** AT\&T, USA
}

\section{Introduction}

We seek to provide an alternative theoretical perspective on concept mapping (a formalism for representing structural knowledge) to that provided by Ray McAleese in this issue of ALT-J (auto-monitoring). We begin with an overview of concept maps as a means of describing a learner's knowledge constructs, and then discuss a broader class of tools, Mindtools, of which concept maps are a member. We proceed by defining Mindtools as formalisms for representing knowledge, and further elaborate on concept maps as a formalism for representing a particular kind of knowledge: structural knowledge. We then address McAleese's use of the term auto-monitoring and some of the steps in his model of concept maps. Finally, we describe some limitations of concept mapping as a formalism and as a cognitive learning strategy.

Concept maps, both in how they are used and theoretically based, touch some of the central elements of instructional technology and learning. Concept maps address the critical issue of how we organize our knowledge structures within and between domains. They, in some sense, model how we represent and apply knowledge. Assuming that instructional technology, as a field, has a goal of being able systematically to make instruction more effective, then tools 